\title{
Neural Correlates of Active Avoidance Behavior in Superior Colliculus
}

\author{
Jeremy D. Cohen and Manuel A. Castro-Alamancos \\ Department of Neurobiology and Anatomy, Drexel University College of Medicine, Philadelphia, Pennsylvania19129
}

Active avoidance of harmful situations seems highly adaptive, but the underlying neural mechanisms are unknown. Rats can effectively use the superior colliculus during active avoidance to detect a salient whisker conditioned stimulus (WCS) that signals an aversive event. Here, we recorded unit and field potential activity in the intermediate layers of the superior colliculus of rats during active avoidance behavior. During the period preceding the onset of the WCS, avoids are associated with a higher firing rate than escapes (unsuccessful avoids), indicating that a prepared superior colliculus is more likely to detect the WCS and lead to an avoid. Moreover, during the WCS, a robust ramping up of the overall firing rate is observed for trials leading to avoids. The firing rate ramping is not caused by shuttling and may serve to drive downstream circuits to avoid. Therefore, a robust neural correlate of active avoidance behavior is found in the superior colliculus, emphasizing its role in the detection of salient sensory signals that require immediate action.

\section{Introduction}

Active avoidance is a learning paradigm in which the subject learns to avoid an aversive event by producing an appropriate behavioral response (avoid) during an interval signaled by the presentation of a conditioned stimulus (CS) (Mowrer, 1960; Bouton, 2007). Avoidance seems useful to eliminate harmful situations but can be maladaptive, such as during anxiety disorders (phobias). Despite the relevance, little is known about the neural circuitry involved in active avoidance behavior.

Rats readily learn to avoid an aversive stimulus by detecting a $10 \mathrm{~Hz}$ electrical CS applied unilaterally to the whisker pad (Castro-Alamancos, 2004a; Cohen and Castro-Alamancos, 2007); note that in our study, CS detection is operationally defined as a successful avoid. The whisker pad CS (WCS) travels to the trigeminal complex in the brainstem from where it is distributed to two main targets, the superior colliculus in the midbrain tectum (trigeminotectal pathway), and the somatosensory thalamus (trigeminothalamic pathway), which relays the WCS to the barrel cortex (Cohen and Castro-Alamancos, 2007). Moreover, the WCS reaching the barrel cortex returns to the superior colliculus via corticotectal fibers, producing a characteristic electrophysiological response in the intermediate layers of the superior colliculus consisting of two successive peaks (Cohen et al., 2008); peak 1 is mediated by trigeminotectal inputs, and peak 2 is mediated by corticotectal activity returning from the barrel cortex.

The superior colliculus is well suited to mediate active avoidance because it forms extensive descending projections that give rise to orienting and escape responses (Sprague and Meikle, 1965;

\footnotetext{
Received March 24, 2010; revised May 6, 2010; accepted May 12, 2010.

This work was supported by the National Institutes of Health.

Correspondence should be addressed to Dr. Manuel Castro-Alamancos, Department of Neurobiology and Anatomy, Drexel University College of Medicine, 2900 Queen Lane, Philadelphia, PA 19129. E-mail:mcastro@ drexelmed.edu.

D0I:10.1523/JNEUROSCI.1497-10.2010

Copyright $\odot 2010$ the authors $\quad 0270-6474 / 10 / 308502-10 \$ 15.00 / 0$
}

Schneider, 1969; Sparks, 1986; Dean et al., 1989; Westby et al., 1990; Redgrave et al., 1993; Stein and Meredith, 1993; Brandao et al., 1994), and because it forms ascending loops with the basal ganglia (Comoli et al., 2003; Jiang et al., 2003; Dommett et al., 2005; McHaffie et al., 2005; Redgrave and Gurney, 2006), a set of structures that may be involved in active avoidance (Delacour et al., 1977; Chavez-Martinez et al., 1987; Packard and Knowlton, 2002). Cells in the superior colliculus also respond to noxious stimulation (Stein and Dixon, 1979; McHaffie et al., 1989; Redgrave et al., 1996a,b), such as that conveyed by a footshock during active avoidance conditioning. Thus, in principle, the WCS and footshock can be associated in this structure. Recent lesion studies have shown that the superior colliculus can mediate active avoidance behavior to a WCS on its own, in the absence of the somatosensory thalamus and the corticotectal activity driven by the WCS (Cohen and Castro-Alamancos, 2007), as long as the WCS is psychophysically salient (Cohen and CastroAlamancos, 2010), such as the one used here. Therefore, the superior colliculus can serve as an early relay station for rapid detection of sensory signals that are behaviorally significant and require immediate action, an early sensorimotor hub well suited to mediate active avoidance. Here, we recorded neural activity simultaneously from the superior colliculus and barrel cortex of animals performing an active avoidance task to determine whether neural activity differs between avoids, escapes (unsuccessful avoids), and spontaneous shuttling.

\section{Materials and Methods}

Nine adult male Sprague Dawley rats (225-250 g) were used. Animals were cared for in accordance with National Institutes of Health guidelines for laboratory animal welfare. All experiments were approved by the Drexel University Institutional Animal Care and Use Committee. At all times, food and water were available ad libitum. All animals were initially housed in groups of three for the first block of active avoidance training sessions. Once the animals were assigned to an experimental group, they were housed individually for the remainder of the experimental protocol. 
Surgical procedures. For all recovery surgeries, animals were anesthetized with sodium pentobarbital (50 mg/kg, i.p.) and placed in a stereotaxic frame. All skin incisions and frame contacts with the skin were injected with lidocaine (2\%). Throughout the surgery, body temperature was automatically maintained constant with a heating pad (Harvard Apparatus). During recovery from surgery, animals received a dose of buprenorphine $(0.03 \mathrm{mg} / \mathrm{kg}, \mathrm{i} . \mathrm{m}$.) to reduce pain every $12 \mathrm{~h}$ for $3 \mathrm{~d}$. Recovery from whisker pad electrode and microelectrode implantations involved $7 \mathrm{~d}$ before retesting.

Whisker pad electrode implantation. Before training in the WCS task (see below), an insulated stainless steel bipolar electrode was placed in the left whisker pad subcutaneously to stimulate the whisker pad (CastroAlamancos, 2004a). Electrode pole separation was $\sim 1 \mathrm{~mm}$. The wires were normally placed around whiskers $\mathrm{C} 2-\mathrm{C} 4$. All electrodes and connectors were held in place using miniscrews and dental cement on the skull.

Active avoidance training. Animals were trained in the active avoidance task using procedures similar to those described previously (CastroAlamancos, 2004a; Cohen and Castro-Alamancos, 2007). We use two versions of the active avoidance task depending on the CS that is used. The auditory version uses an auditory CS, and the whisker version uses a WCS. Animals are placed in a standard shuttle box controlled using MedPC software (MED Associates) that consists of two compartments separated by a partition extending up from the grid floor that the animal has to traverse to shuttle between compartments. A single training trial consisted of a $7 \mathrm{~s}$ avoidance interval followed by a $10 \mathrm{~s}$ escape interval. During the avoidance interval, a CS was presented for the duration of the interval or until the animal produced a conditioned response by moving to the adjacent compartment (avoid), whichever occurred first. If the animal avoided, the CS was terminated, and no escape interval was presented. However, if the animal did not avoid, then during the escape interval, a mild scrambled electric footshock (unconditioned stimulus, 0.3-0.6 mA) was delivered through the grid floor of the occupied half of the shuttle box. This mild electrical footshock motivates the animal to move readily to the adjacent compartment (escape), at which point the footshock and CS are coterminated, ending the trial. During the intertrial interval, the animal awaited the next trial and was free to cross between compartments at will. These spontaneous responses are called intertrial crossings. The duration of the intertrial interval in the avoidance task was determined by the investigator. Trials were triggered by the experimenter to assure that the animal was not "distracted" during the presentation of the CS. In particular, the investigator would start a trial as long as the animal was not grooming or producing an intertrial crossing, and after at least $15 \mathrm{~s}$ had passed since the previous trial. The recorded variables that represent task performance are the number of avoids and the latency of avoids (from the CS onset).

For all animals, training began with the auditory version of the task, which uses an auditory CS ( $8 \mathrm{kHz}, 82 \mathrm{~dB}$ tone). Training was conducted over three or four 50-trial sessions (one session per day) until a high level of consistent auditory CS-mediated avoidance behavior was produced ( $>70 \%$ avoidance rate). Afterward and before training in the WCS version of the task, animals were subjected to unilateral implantation of a whisker pad stimulating electrode as described above. All animals were further trained in the WCS version of the active avoidance task. The WCS consisted of a $10 \mathrm{~Hz}$ (1 ms duration) electrical stimulus train delivered through two wires implanted under the skin of the whisker pad (CastroAlamancos, 2004a; Cohen and Castro-Alamancos, 2007). The WCS stimulus was set at intensity just below that resulting in subtle movement of a few (three to five) whiskers but no elicitation of muscle twitches $(0.25-0.60 \mathrm{~mA})$. On the first day of training in the WCS task, animals were placed in the training apparatus for an acclimation session, during which the appropriate WCS intensity was determined for each animal. Training was conducted over four 50 -trial sessions.

Chronic electrophysiology. Animals were implanted with a whisker pad stimulating electrode and two recording electrodes at the same time. The recording electrodes were aligned with the whisker pad stimulating electrode by recording evoked responses during surgery. Once in place, the electrodes attached to head connectors were fixed to the skull by screws and dental cement. In all animals, a field potential (FP) electrode was implanted in the barrel cortex [bregma (in mm): anteroposterior (A/P), -2.7 ; mediolateral $(\mathrm{M} / \mathrm{L}), 5.0$; dorsoventral (D/V), 0.5-1.0]. The FP electrodes consisted of blunt insulated stainless steel wires $(100 \mu \mathrm{m}$ outer diameter, $\sim 0.5 \mathrm{M} \Omega$ ). Concurrently, either an FP electrode or a multiunit activity (MUA) electrode was implanted in the superior colliculus (lambda: A/P, 2.2; M/L, 2.2; D/V , 4-4.5). The MUA electrode consisted of a higher-impedance insulated tungsten electrode edged to a fine tip (100 $\mu \mathrm{m}$ outer shaft diameter, 2-7 M $\Omega$ ). The reference electrode consisted of an FP electrode placed above the superior colliculus, and the ground was attached to skull screws. In some recording sessions, clear single-unit activity was recorded through the MUA electrode, but we combined these cases with MUA sessions. Also, in some cases, FP activity was also collected in the superior colliculus through the MUA recording electrode.

During recording sessions, the head connector was attached to a headstage operational amplifier with unity gain that lead through fine tether cables to an electrical swivel that terminated in the amplifiers and recording system. During electrophysiological recording sessions (one per day), the animal was subjected to training in the WCS active avoidance task in a shuttle box as described above. During the sessions, electrophysiological activity was continuously recorded in synchrony with digital video of the behavior (Cineplex; Plexon).

Measures and statistical analyses. For electrophysiological results, during the active avoidance task, we measured different FP and MUA responses, and data points correspond to recording sessions on different days. Before WCS, we measured neural activity (FP and MUA) during a $2 \mathrm{~s}$ period preceding WCS onset in each trial (pre-WCS). During the WCS, we measured the neural responses evoked by each stimulus in the $10 \mathrm{~Hz}$ WCS train during specific response windows after stimulus. Thus, in barrel cortex, we measured the peak amplitude and time to peak (peak latency) of FP responses in barrel cortex during a 5-30 ms window after stimulus. In superior colliculus, we measured the peak amplitude of two different FP responses that encompass different time windows: peak 1 (3-8 ms) and peak2 (9-20 ms). Also in superior colliculus, we measured MUA as the number of spikes evoked per stimulus for three time windows corresponding to peak1 (3-8 ms), peak2 (9-20 ms), and peak3 (21-90 ms), or we used a large time window that encompasses the sum of the three peaks (3-90 ms after stimulus), which we call peak1-3. The border between peak 1 and peak 2 was slightly adjusted per animal (range, 1-3 ms) based on the evoked responses.

Statistical analyses for electrophysiological responses measured during the active avoidance task began by conducting two-factor repeatedmeasures ANOVAs of the effect of avoidance (avoids vs escapes) and the effect of stimulus number from WCS onset (first 10 stimuli in a WCS) or from WCS offset (last 10 stimuli before avoid). Note that for the escapes, the last 10 stimuli from WCS offset correspond to the 10 last stimuli before the footshock. Significant main effects were decomposed by pairwise comparisons that were either parametric (Tukey's), if the two groups involved were normally distributed (according to the ShapiroWilk normality test), or nonparametric (Wilcoxon signed ranks). The $p$ values of the multiple-comparisons tests were adjusted by the number of comparisons.

\section{Results \\ Dataset}

We recorded neural activity within the barrel cortex and superior colliculus as animals performed the WCS active avoidance task (see Materials and Methods). Activity in barrel cortex was monitored with an FP electrode ( $n=9$ animals), and activity in superior colliculus was monitored with an FP electrode $(n=3$ animals) or a MUA electrode ( $n=6$ animals), from which we also obtained some FP data. FP activity reflects primarily local synchronous subthreshold (mostly synaptic) neural events, whereas MUA reflects the suprathreshold (spike) activity of cell clusters surrounding the electrode. Simultaneous FP and intracellular recordings from individual whisker-responsive cells in the intermediate layers of superior colliculus have shown that the FP 


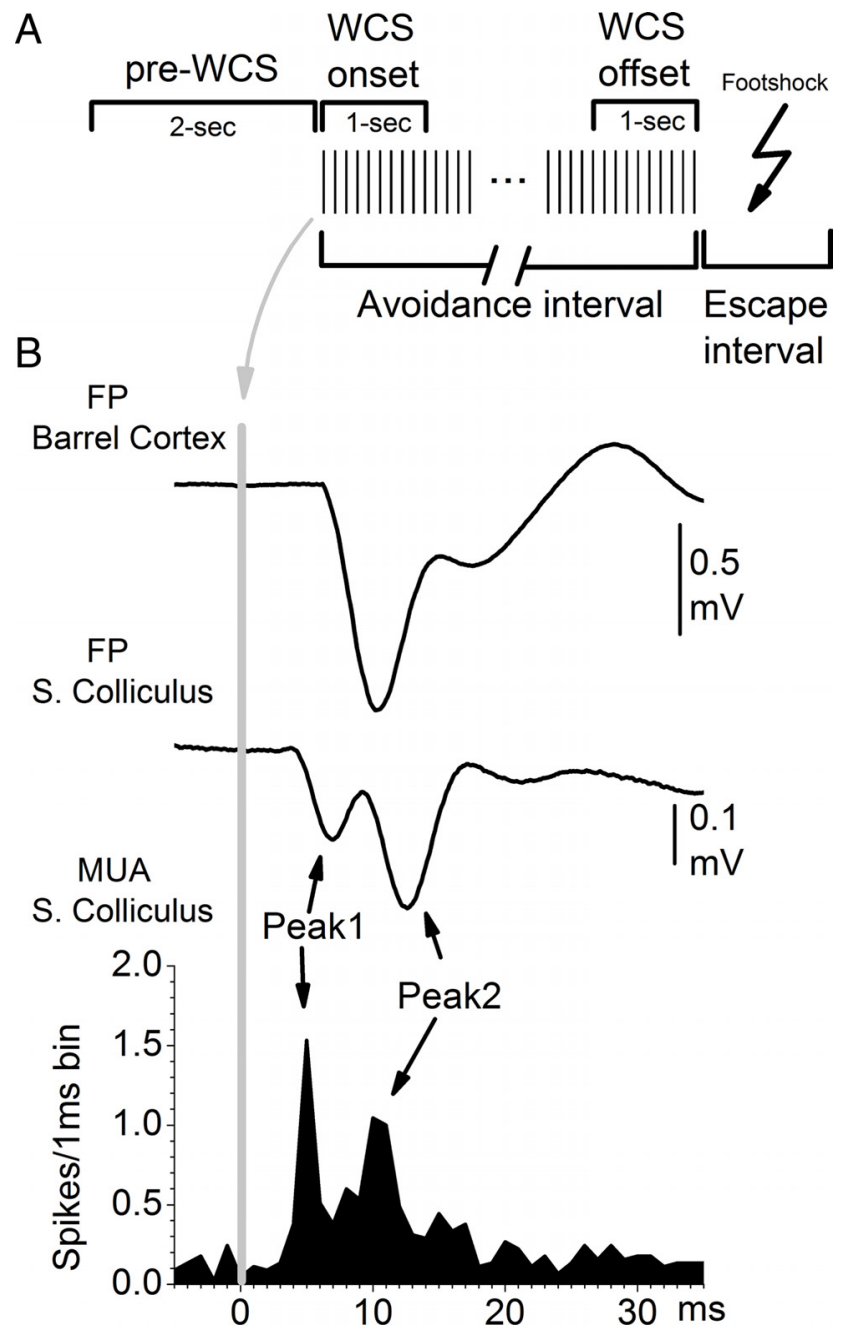

Figure 1. Schematic representation of pre-WCS and WCS periods measured during each trial and typical FP and MUA responses. $A$, Two seconds of pre-WCS activity was measured to determine the state of barrel cortex and superior colliculus before each trial. During the avoidance interval, the WCS consisted of a $10 \mathrm{~Hz}$ train delivered to the whisker pad. Measurements were done on the 10 first stimuli (WCS onset) and the last 10 stimuli (WCS offset) during the avoidance interval. $\boldsymbol{B}$, Typical FP (barrel cortex and superior colliculus) and MUA [superior colliculus (S. Colliculus)] responses evoked by each stimulus in the WCS train. Peak1 and Peak2 FP and MUA responses in superior colliculus are pointed by arrows. Each response shown is the average of 30 trials and corresponds to the first stimulus in the WCS train.

responses driven by whisker stimulation reflect closely the intracellular synaptic responses (Cohen et al., 2008).

The animals were trained in the WCS avoidance task for four sessions ( 50 trials per session) during which they learned to successfully avoid the presentation of a mild aversive stimulus (footshock) by detecting the WCS. For each WCS trial in the task, several measurements were performed on FP and MUA in barrel cortex and superior colliculus. Measurements were of pre-WCS and WCS activity (Fig. $1 A$ ). The pre-WCS activity refers to FP or MUA spontaneous activity preceding each WCS presentation by $2 \mathrm{~s}$. The WCS activity refers to FP and MUA responses evoked by each stimulus in the WCS $10 \mathrm{~Hz}$ train. Because trials last different periods of time depending on when and if the animal avoided, the responses evoked by the first 10 WCS in a trial (from WCS onset) and the responses evoked by the last 10 WCS during the avoidance interval in a trial (from WCS offset) were measured. Because of the large artifacts, neural responses were not measured during footshock presentations during the escape interval. Figure $1 B$ shows typical FP (barrel cortex and superior colliculus) and MUA (superior colliculus) responses evoked by each stimulus in the WCS train.

We conducted two main analyses on this dataset to compare electrophysiological responses during avoids and escapes. The first analysis compared the escapes and avoids from all the trials in all the sessions (all trials). The second analysis compared the "early-session escapes" when the animal is learning the task in the first session to the "late-session avoids" when the animal has mastered the task in the last session and seldom fails to avoid (early-session escapes/late-session avoids). This second analysis reflects how sensory responses evoked by the WCS are transformed by learning the task. During early-session escapes, the animal has not learned to avoid the aversive event by shuttling during the WCS, and thus most trials lead to escapes; the animal is not trained and is generally stressed because it is being shocked often for not avoiding. During late-session avoids, the animal effectively avoids the aversive event by detecting the WCS; the animal is well trained.

\section{Pre-WCS MUA in superior colliculus predicts active avoidance}

There is a large literature relating spontaneous network activity, including cortical oscillations, to the ability to detect sensory stimuli and perform behavioral tasks in several species (for review, see Jensen et al., 2007; Schroeder and Lakatos, 2009). To address the putative relationship between spontaneous neural activity and success in active avoidance behavior, we compared FP and MUA activity preceding each stimulus trial (pre-WCS) between avoids and escapes.

In the barrel cortex, we found that there was no significant difference in the fast Fourier transform power spectrum of preWCS ( 2 s before each trial) FP activity between escapes and avoids when either all trials are considered ( $p=0.6 ; n=28$ sessions) (Fig. $2 A$ ) or when early-session escapes are compared to latesession avoids ( $p=0.9 ; n=7$ animals) (Fig. $2 B$ ). Thus, the power spectrum of spontaneous FP activity in barrel cortex does not predict successful active avoidance performance. In the superior colliculus, we found that there was no significant difference in the fast Fourier transform power spectrum of pre-WCS ( $2 \mathrm{~s}$ before each trial) FP activity between escapes and avoids when either all trials are considered ( $p=0.9 ; n=28$ sessions) (Fig. $2 C$ ) or when early-session escapes are compared to latesession avoids ( $p=0.45 ; n=7$ animals) (Fig. $2 D$ ). However, when individual frequency ranges were compared, we found that the $5-10 \mathrm{~Hz}$ (theta) range was significantly stronger for latesession avoids than for early-session escapes $(p<0.05)$. Although this difference was small and not significant when all trials were considered, it may indicate that theta frequency range oscillations are more robust in well trained animals performing the task correctly. The source of these theta oscillations is unclear, but there is no evidence that they originate in superior colliculus. Most likely, this activity is volume conducted from the hippocampal/cortical areas overlying the superior colliculus, which are known to produce robust theta oscillations during active behaviors (Vanderwolf, 1988). In conclusion, the power spectrum of spontaneous FP activity preceding each trial in the barrel cortex or superior colliculus does not predict successful active avoidance performance.

In contrast to FP activity, we found that pre-WCS MUA in superior colliculus is associated with successful active avoidance performance. For the first analysis in which all trials are considered, we found that there was a significant difference in pre-WCS MUA in superior colliculus between escapes and avoids $(p<$ 
A
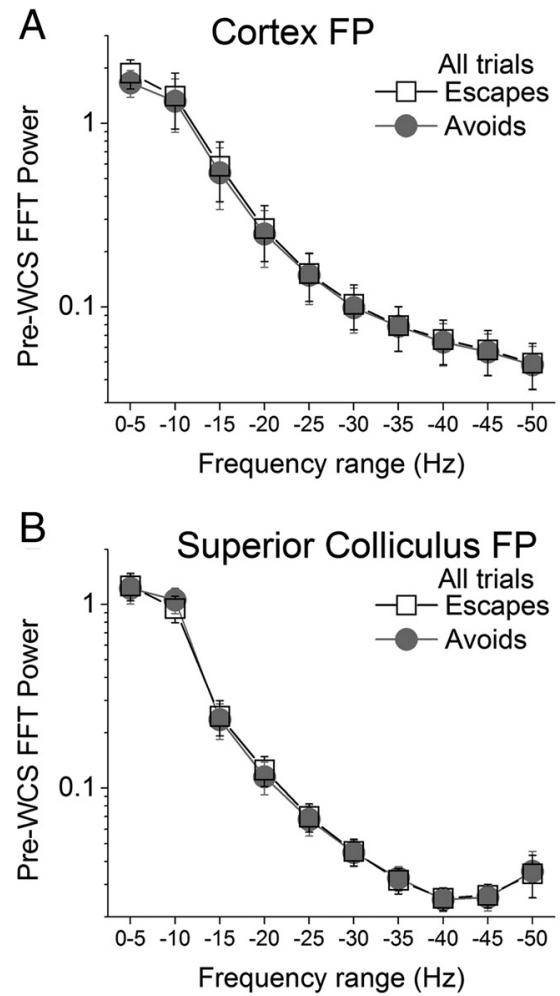
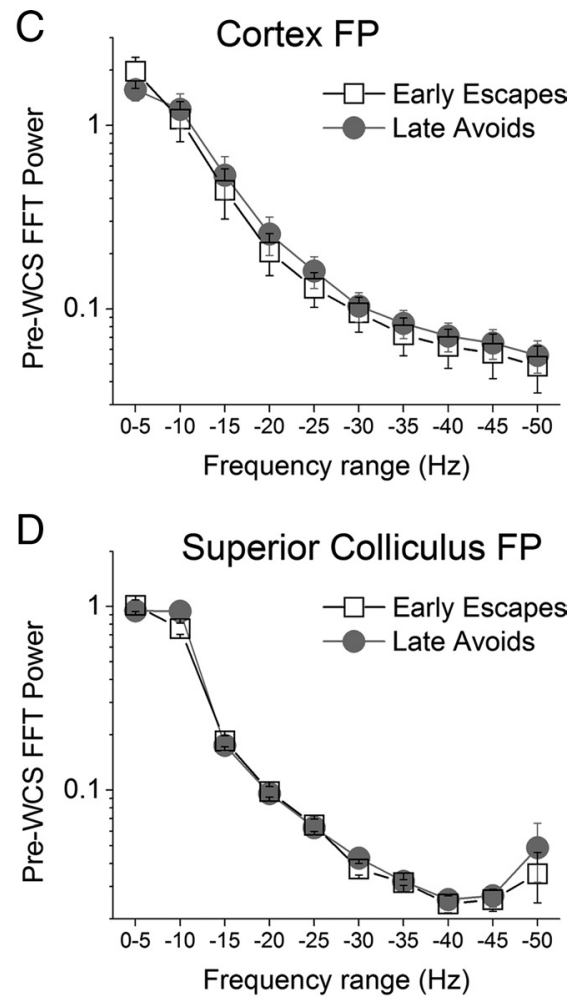

$\mathrm{E}$

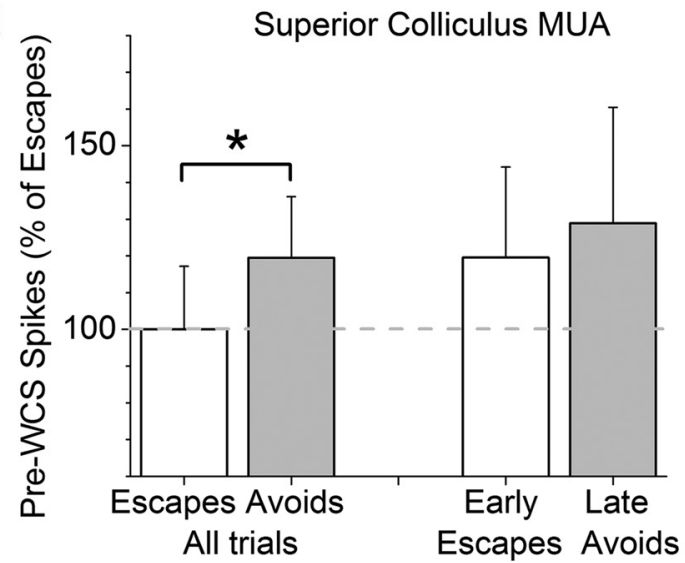

Figure 2. Pre-WCS neural activity and active avoidance. $A, B$, Fast Fourier transform power spectrum of pre-WCS barrel cortex FP activity taken from the period of $2 \mathrm{~s}$ before each WCS trial during trials that lead to escapes and those that lead to avoids for all trials $(\boldsymbol{A})$ and during early-session escapes and late-session avoids trials $(\boldsymbol{B})$. $\boldsymbol{C}, \boldsymbol{D}$, Fast Fourier transform power spectrum of pre-WCS superior colliculus FP activity taken from the period of 2 s before each WCS trial during trials that lead to escapes and those that lead to avoids for all trials $(\boldsymbol{C})$ and during early-session escapes and late-session avoids trials (D).E, Spontaneous pre-WCS MUA in superior colliculus for the period of $2 \mathrm{~s}$ before each WCS trial during trials that lead to escapes and those that lead to avoids for all trials and during early-session escapes and late-session avoids trials. MUA for each group is plotted as a percentage of escapes (All trials). For all trials, MUA preceding the onset of each trial is significantly larger when the trial leads to an avoid than when it leads to an escape. ${ }^{*} p<0.01$. FFT, Fast Fourier transform. Error bars are \pm SEM.

$0.01 ; n=28$ sessions) (Fig. $2 E$ ). MUA during the $2 \mathrm{~s}$ preceding the WCS onset was significantly higher for avoids than for escapes. Thus, the state of spontaneous MUA in superior colliculus predicts whether the animal will detect the WCS or not. For the second analysis in which early-session escapes are compared to late-session avoids, we found that there was no significant difference in pre-WCS MUA between early-session escapes and latesession avoids ( $p=0.8 ; n=7$ animals) (Fig. $2 E$ ). This could be attributable to the high level of MUA in superior colliculus during early-session escapes, which may be related to the higher level of stress during the early learning trials. To determine whether the higher pre-WCS MUA for avoids compared to escapes for all trials reflected a difference in a particular rhythm (frequency range), we calculated an autocorrelation function for the pre-WCS MUA ( $2 \mathrm{~s}$ before trial onset) for avoids and escapes (normalized by the total number of interval counts; -1000 to $1000 \mathrm{~ms}$ range; $1 \mathrm{~ms}$ bin; $n=28$ sessions). This analysis revealed no significant differences in rhythmic firing at any frequency between avoids and escapes. Thus, whereas the pre-WCS firing rate is higher for avoids than for escapes (for all trials), this does not reflect a difference in a particular firing frequency range but an overall change in rate.

In conclusion, spontaneous pre-WCS MUA in superior colliculus is consistently larger during avoids than during escapes, indicating that an active (prepared) superior colliculus is more likely to detect the WCS than an inactive (nonprepared) superior colliculus.

\section{FP responses in barrel cortex during active avoidance}

Neocortex FP responses evoked by afferent inputs are robustly modulated by the behavioral state of the animal (Castro-Alamancos and Connors, 1996; Castro-Alamancos and Oldford, 2002; Castro-Alamancos, 2004a). Here, we determined whether FP responses in barrel cortex driven by the WCS are able to distinguish between avoids and escape trials.

We measured the peak amplitude and latency (5-30 ms after stimulus) of FP responses in barrel cortex evoked by each stimulus during presentation of the $10 \mathrm{~Hz}$ WCS (from onset and offset) for all trials and for early-session escapes compared to late-session avoids. When all trials are considered ( $n=28$ sessions) (Fig. 3), the peak amplitude of FP responses in barrel cortex from WCS onset were not significantly different between escapes and avoids ( $p=0.8$ ) (Fig. 3A). However, responses from WCS offset were significantly more suppressed during avoids than during escapes $(p<$ 0.01) (Fig. 3A).

When early-session escapes and latesession avoids are compared ( $n=7$ animals) (Fig. 4), the peak amplitude of FP responses in barrel cortex from WCS onset were significantly more suppressed during avoids than during escapes $(p<0.01)$ (Fig. 4A). Also, responses from WCS offset were significantly more suppressed during avoids than during escapes $(p<0.01)$ (Fig. 4A). These results indicate that the peak amplitude of adapted barrel cortex responses is able to distinguish between avoids and escapes, so that barrel cortex FP responses are significantly more suppressed during avoids than during escapes.

Barrel cortex responses evoked by whisker stimulation show robust sensory adaptation to frequency (Ahissar, 1998; Chung et 

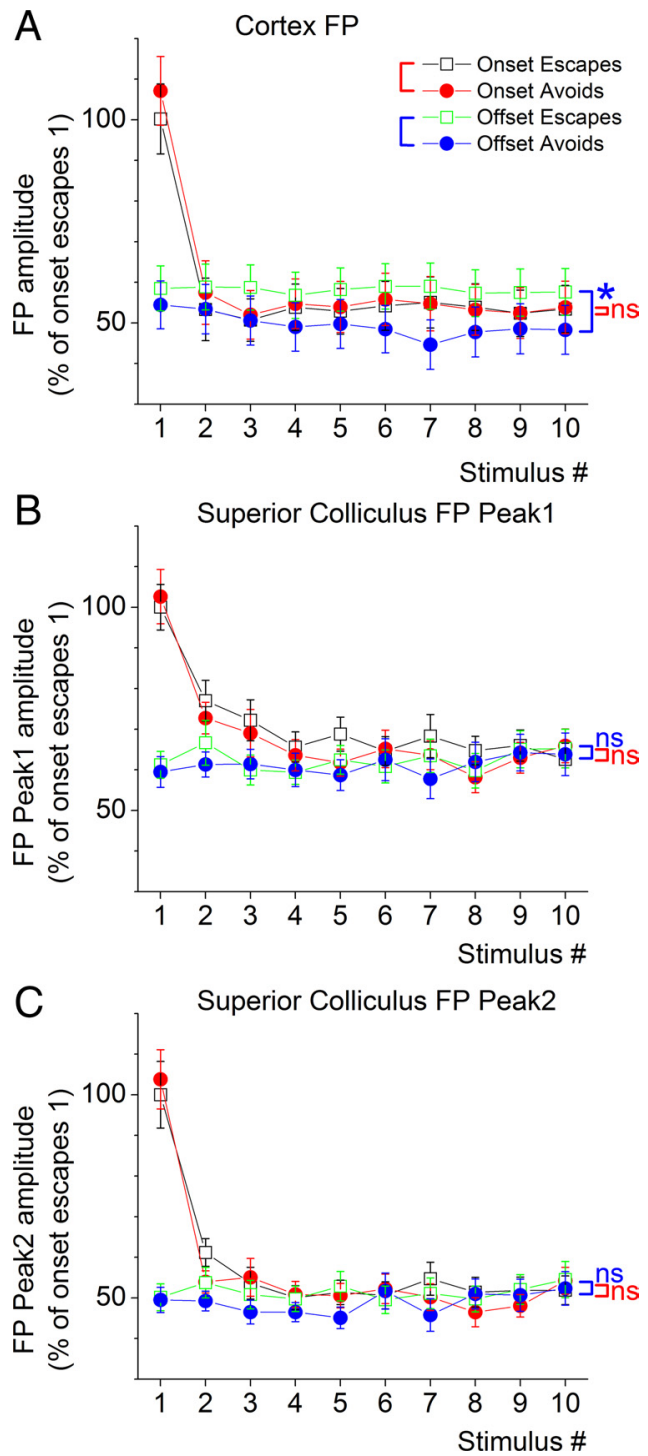

Figure 3. FP activity in barrel cortex and superior colliculus evoked by the WCS during performance in the active avoidance task. Barrel cortex FP peak amplitude responses $(\boldsymbol{A})$ and superior colliculus (SC) FP peak1 $(\boldsymbol{B})$ and FP peak2 $(\boldsymbol{C})$ responses evoked by the WCS $(10 \mathrm{~Hz})$ that lead to either avoids or escapes in the task are shown. The responses are plotted from WCS onset, which includes the first 10 stimuli in the WCS, and from WCS offset, which includes the last 10 stimuli in the WCS, before avoids or before the onset of the escape interval for escapes. Statistically significant differences $(p<0.05)$ between avoids and escapes are marked with brackets on the right. If present, the red bracket and asterisk indicate a significant difference between avoids and escapes from WCS onset, and the blue bracket and asterisk indicate a significant difference between avoids and escapes from WCS offset. ns, Not significant. $p>$ 0.05. Error bars are \pm SEM.

al., 2002; Garabedian et al., 2003; Castro-Alamancos, 2004b; Khatri et al., 2004; Moore, 2004), and adaptation is highly dependent on the state of the animal (Castro-Alamancos and Oldford, 2002; Castro-Alamancos, 2004a). Regarding adaptation of FP responses in barrel cortex, there was significant adaptation between S1 and S10 for avoids and escapes from WCS onset when all trials are considered $(p<0.01)$ and when only early-session escapes and late-session avoids are considered. Regarding the time to peak of FP responses in barrel cortex, it was less affected than the peak amplitude. In particular, there was no significant difference between avoids and escapes from WCS onset ( $p=0.7)$ or from WCS offset $(p=0.7)$ when all trials are considered, or when
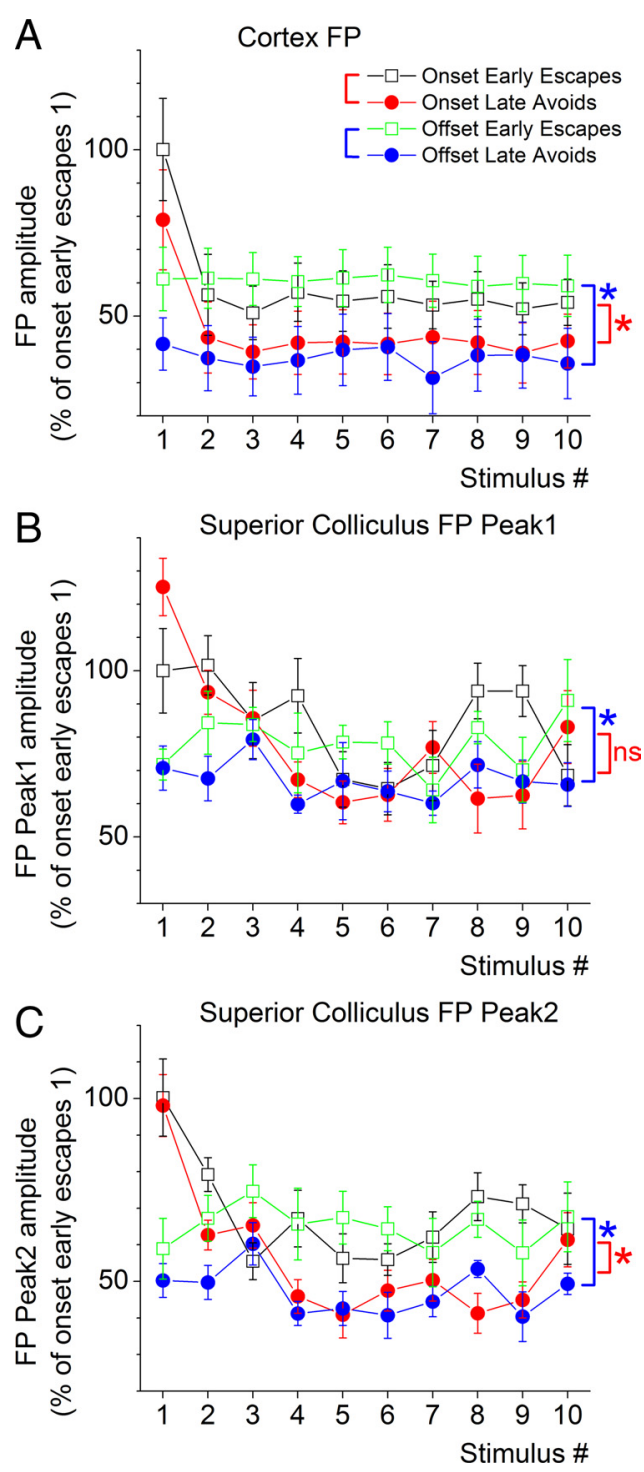

Figure 4. FP activity in barrel cortex and superior colliculus evoked by the WCS during performance in the active avoidance task comparing early-session escapes and late-session avoids. Barrel cortex FP peak amplitude responses $(\boldsymbol{A})$ and superior colliculus FP peak1 $(\boldsymbol{B})$ and FP peak2 $(C)$ responses evoked by the WCS $(10 \mathrm{~Hz})$ for early-session escapes and late-session avoids are shown. The responses are plotted from WCS onset, which includes the first 10 stimuli in the WCS, and from WCS offset, which includes the last 10 stimuli in the WCS, before late-session avoids or before the onset of the escape interval for early-session escapes. Statistically significant differences $(p<0.05)$ between late-session avoids and early-session escapes are marked with vertical brackets on the right. If present, the red bracket and asterisk indicate a significant difference between late-session avoids and early-session escapes from WCS onset, and the blue bracket and asterisk indicate a significant difference between late-session avoids and earlysession escapes from WCS offset. ns, Not significant. $p>0.05$. Error bars are \pm SEM.

late-session avoids and early-session escapes from WCS onset ( $p=0.1)$ or from WCS offset $(p=0.75)$ are considered.

In conclusion, when all trials of all sessions are considered, FP responses in barrel cortex from WCS onset cannot distinguish avoids from escapes, but FP responses in barrel cortex from WCS offset are more suppressed during avoids than during escapes. When early-session escapes and late-session avoids are compared, FP responses from WCS onset and from WCS offset are both more suppressed during late-session avoids than during early-session escapes. Thus, sensory suppression in barrel cortex is associated with successful active avoidance. 
A
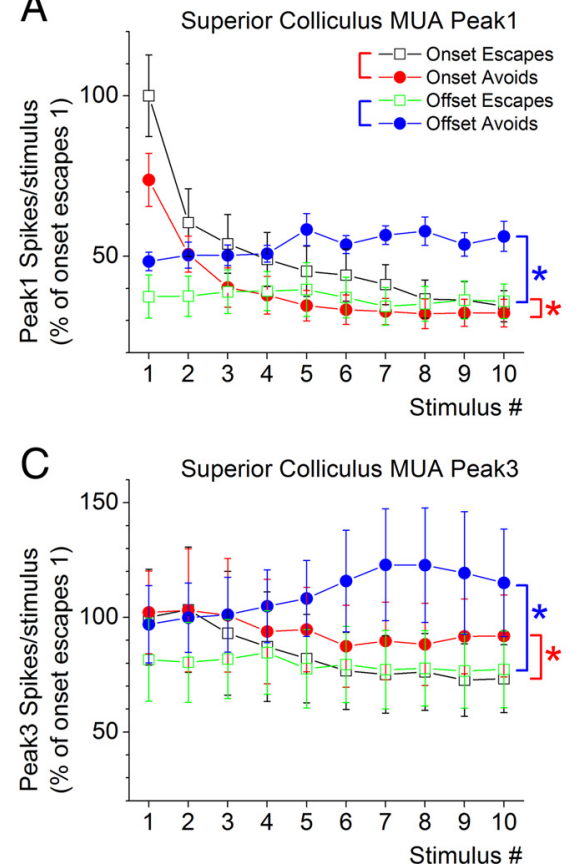

B

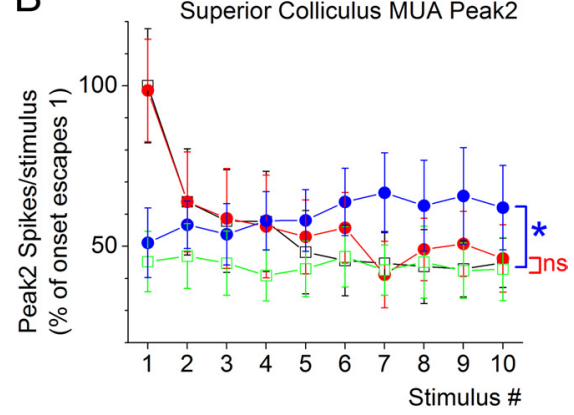

D

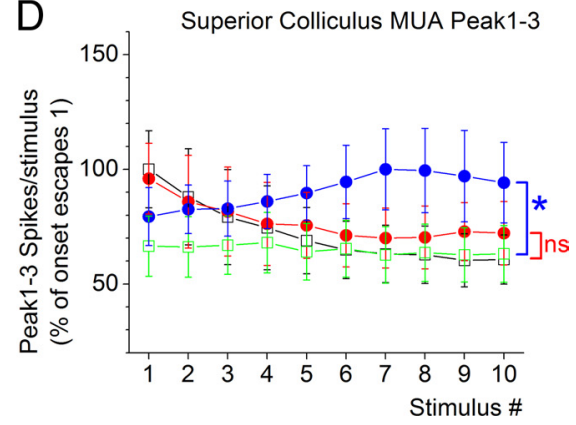

Figure 5. MUA in superior colliculus evoked by the WCS during performance in the active avoidance task. Superior colliculus MUA peak1 $(\boldsymbol{A})$, peak2 $(\boldsymbol{B})$, peak3 $(\boldsymbol{C})$, and peak1-3 $(\boldsymbol{D})$ responses evoked by the WCS $(10 \mathrm{~Hz})$ that lead to either avoids or escapes in the task are shown. The responses are plotted from WCS onset, which includes the first 10 stimuli in the WCS, and from WCS offset, which includes the last 10 stimuli in the WCS, before avoids or before the onset of the escape interval for escapes. Statistically significant differences $(p<0.05)$ between avoids and escapes are marked with vertical brackets on the right. If present, the red bracket and asterisk indicate a significant difference between avoids and escapes from WCS onset, and the blue bracket and asterisk indicate a significant difference between avoids and escapes from WCS offset. ns, Not significant. $p>0.05$. Error bars are \pm SEM.

FP responses in superior colliculus during active avoidance FP responses evoked in superior colliculus by whisker stimulation shows a characteristic waveform comprising two successive peaks (at $\sim 4$ and $\sim 12 \mathrm{~ms}$ after stimulus) (Fig. 1) that are driven by trigeminotectal (peak1) and corticotectal (peak2) inputs (Cohen et al., 2008). Although rats can use these responses to produce successful active avoidance behavior (Cohen and CastroAlamancos, 2007), it is not known how these responses vary as a function of active avoidance behavior. Here, we determined whether FP responses in superior colliculus driven by the WCS are able to distinguish between avoids and escape trials.

We measured the peak amplitude of peak1 (3-9 ms after stimulus) and peak2 (10-25 ms after stimulus) FP responses in superior colliculus evoked by each stimulus during presentation of the $10 \mathrm{~Hz}$ WCS (from onset and offset) for all trials and for earlysession escapes compared to late-session avoids. When all trials are considered ( $n=25$ sessions) (Fig. 3 ), the amplitude of peak1 and the peak2 FP responses in superior colliculus from WCS onset were not significantly different between escapes and avoids ( $p=0.16$ and $p=0.5$, respectively) (Fig. $3 B, C$ ). This was also the case for peak1 and peak2 responses from WCS offset $(p=0.4$ and $p=0.06$, respectively) (Fig. $3 B, C$ ).

When early-session escapes and late-session avoids are compared ( $n=7$ animals) (Fig. 4 ), there was no significant difference between avoids and escapes for peak1 ( $p=0.2 ; n=7)$ (Fig. $4 B$ ) $\mathrm{FP}$ responses, but there was for peak2 FP responses in superior colliculus, which were more suppressed during avoids $(p<0.01)$ (Fig. 4C). Also, for the responses from WCS offset, there was a significant difference between avoids and escapes for both peak1 $(p<0.01)$ (Fig. $4 B)$ and peak2 $(p<0.01)$ (Fig. 4C) FP responses in superior colliculus. In both cases, responses were more suppressed during avoids.

Regarding adaptation of FP responses in superior colliculus, there was significant adaptation between S1 and S10 for avoids and escapes from WCS onset for peak 1 and peak 2 responses when all trials are considered $(p<0.01)$ and when only early-session escapes and late-session avoids are considered.

In conclusion, when all trials of all sessions are considered, peak1 and peak2 FP responses in superior colliculus from WCS onset or offset cannot distinguish avoids from escapes. However, when early-session escapes and late-session avoids are compared, peak $1 \mathrm{FP}$ response in superior colliculus from WCS offset and peak2 FP responses from either WCS onset or offset are more suppressed during late-session avoids than during earlysession escapes. Thus, corticotectal (peak2) responses in superior colliculus reflect the cortical sensory suppression when the animal is well trained (latesession avoids).

\section{MUA responses in superior colliculus during active avoidance}

As already mentioned, superior colliculus responses evoked by whisker stimulation produce characteristic peak1 and peak2 responses that are well reflected in single-cell or MUA firing. Here, we determined whether MUA responses in superior colliculus driven by the WCS are able to distinguish between avoids and escape trials. In addition to the peak 1 and peak 2 response windows, we also measured a peak3 window, which encompasses the period between peak 2 to about the next stimulus in the WCS. Moreover, to determine the effect of the WCS on the overall firing, we combined all three response windows into a single window (peak1-3).

We measured the number of spikes per stimulus of peak1 (3-9 ms after stimulus), peak2 (10-20 ms after stimulus), peak3 (21-90 ms after stimulus), and peak1-3 (3-90 ms after stimulus) MUA responses in superior colliculus evoked by each stimulus during presentation of the $10 \mathrm{~Hz}$ WCS (from onset and offset) for all trials and for early-session escapes compared to late-session avoids. When all trials are considered ( $n=25$ sessions) (Fig. 5), MUA from WCS onset was significantly suppressed during avoids compared to escapes for peak1 $(p<0.01 ; n=28)$ responses, but was enhanced for peak3 $(p<0.01)$ responses and unchanged for peak2 $(p=0.3)$ and peak $1-3(p=0.3)$ responses. Thus, peak1 responses from WCS onset are suppressed during avoids compared to escapes, whereas peak3 responses are enhanced. MUA from WCS offset was significantly enhanced during avoids than during escapes for peak $1(p<0.01)$, peak $2(p<$ $0.01)$, peak3 $(p<0.01)$, and peak1-3 $(p<0.01)$ responses (Fig. 5 ). Thus, MUA in superior colliculus from WCS offset is generally enhanced during avoids compared to escapes. Finally, there was significant adaptation between S1 and S10 for avoids and escapes from WCS onset for peak1 and peak2 responses $(p<$ $0.01)$, but not for peak3 responses. 

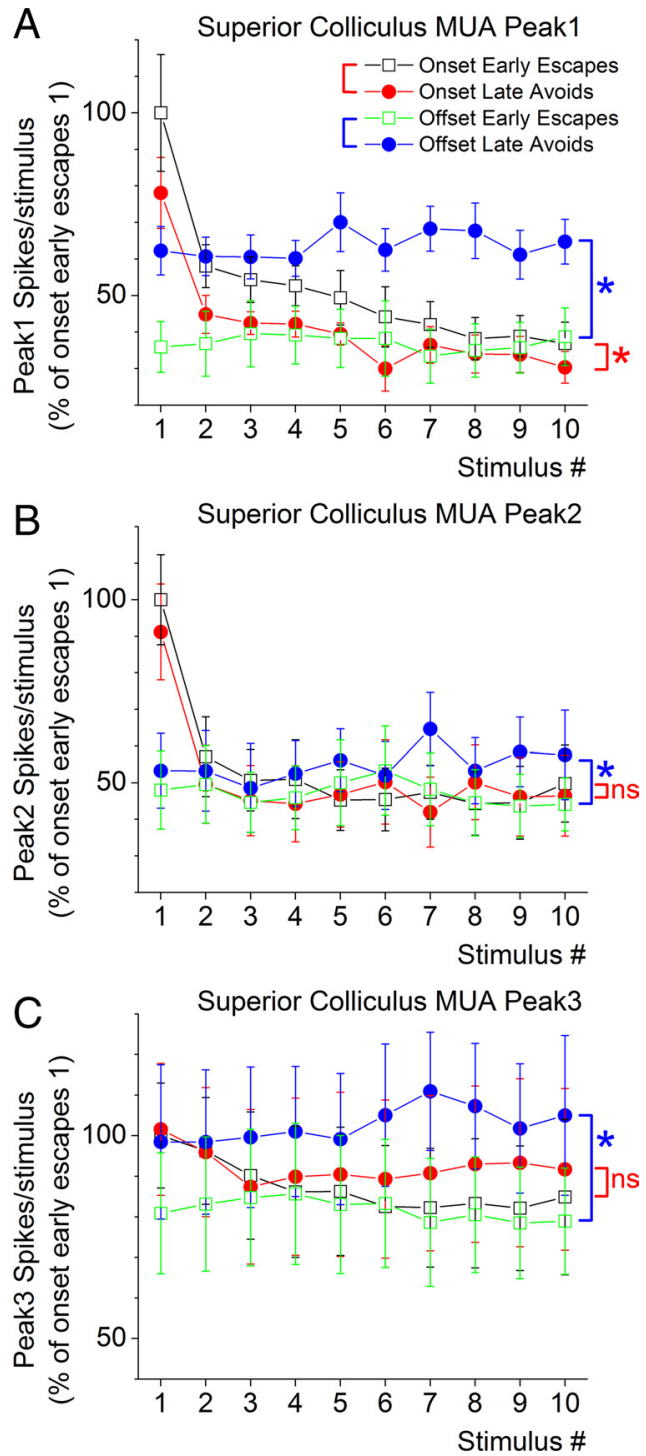

Figure 6. MUA in superior colliculus evoked by the WCS during performance in the active avoidance task comparing early-session escapes and late-session avoids. Superior colliculus MUA peak1 $(\boldsymbol{A})$, peak2 $(\boldsymbol{B})$, and peak3 $(\boldsymbol{C})$ responses evoked by the WCS $(10 \mathrm{~Hz})$ that lead to either late-session avoids or early-session escapes in the task are shown. The responses are plotted from WCS onset, which includes the first 10 stimuli in the WCS, and from WCS offset, which includes the last 10 stimuli in the WCS, before late-session avoids or before the onset of the escape interval for early-session escapes. Statistically significant differences $(p<0.05)$ between late-session avoids and early-session escapes are marked with vertical brackets on the right. If present, the red bracket and asterisk indicate a significant difference between latesession avoids and early-session escapes from WCS onset, and the blue bracket and asterisk indicate a significant difference between late-session avoids and early-session escapes from WCS offset. ns, Not significant. $p>0.05$. Error bars are \pm SEM.

When early-session escapes and late-session avoids are compared ( $n=7$ animals) (Fig. 6), MUA from WCS onset was significantly suppressed during late-session avoids than earlysession escapes for peak1 $(p<0.01)$ but not for peak2 $(p=0.5)$ and peak3 $(p=0.3)$ responses. MUA from WCS offset was significantly enhanced during late-session avoids than during earlysession escapes for peak1 $(p<0.01)$, peak2 $(p<0.05)$, and peak3 $(p<0.01)$ responses. Thus, the MUA responses in superior colliculus from WCS offset are clearly able to distinguish between late-session avoids and early-session escapes, so that late-session avoids produce significantly larger MUA. Finally, there was significant adaptation between S1 and S10 for late-

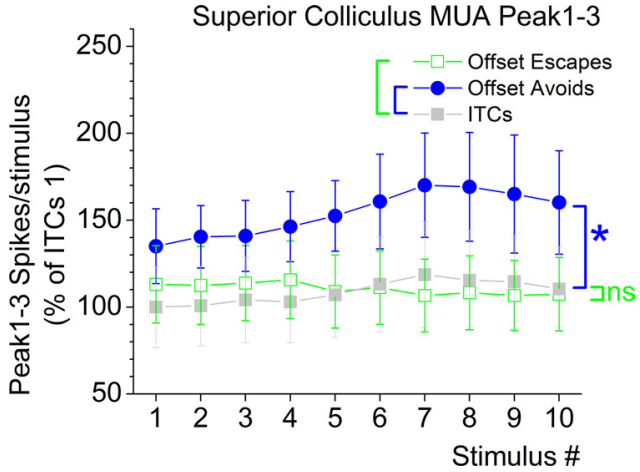

Figure 7. Enhanced MUA in superior colliculus during avoids is not caused by shuttling. Superior colliculus peak1-3 (3-90 ms) MUA evoked by the WCS $(10 \mathrm{~Hz})$ from WCS offset comparing trials that lead to avoids or escapes in the task with the same measurements taken from intertrial crossings (ITCS), when the animal spontaneously shuttles in the cage without a WCS, is shown. The responses are plotted from WCS offset, which includes the last 10 stimuli in the WCS for avoids, escapes, and ITCs. Statistically significant differences $(p<0.05)$ are marked with vertical brackets on the right. The blue bracket and asterisk indicate a significant difference between avoids and ITCS. ns, Not significant. $p>0.05$. Error bars are \pm SEM.

session avoids and early-session escapes from WCS onset for peak1 and peak2 responses $(p<0.01)$.

In conclusion, when all trials of all sessions are considered, peak1, peak2, and peak 3 (and the sum of these, peak1-3) MUA responses from WCS offset easily distinguish avoids and escapes so that activity is larger during avoids. However, only peak 1 and peak3 responses from WCS onset are different during avoids than during escapes. But peak1 responses are more suppressed during avoids than during escapes, whereas peak3 responses are larger during avoids. When early-session escapes and late-session avoids are compared, peak1, peak2, and peak 3 MUA responses from WCS offset also easily distinguish late-session avoids and early-session escapes so that activity is larger during late-session avoids. However, peak1 responses from WCS onset are, again, more suppressed during avoids. Thus, it appears that trigeminotectal (peak1) responses become deemphasized, whereas higherorder (longer-latency) responses increase sharply when animals are successful active avoiders.

\section{Shuttling does not explain the larger MUA during avoids}

The previous results indicate that during avoids, MUA measured from WCS offset in superior colliculus is larger than during escapes for all response time windows. Because the WCS offset data for avoids marks when the animal shuttles, we tested whether the increased MUA depended on the animal shuttling. Thus, for all sessions $(n=28)$, we compared the MUA in superior colliculus during avoids and escapes with the MUA in the same sessions produced by spontaneous intertrial crossings. During intertrial crossings, animals spontaneously shuttle in the cage, but this is not motivated by the WCS, which is not present. During escapes, the WCS is present, but the animal does not shuttle during the avoidance interval, and during avoids, the WCS is present and the animal shuttles. We found that MUA for peak1 $(p<0.01)$, peak2 $(p<0.01)$, peak3 $(p<0.01)$, and peak1-3 $(p<0.01)$ (Fig. 7) during avoids was significantly larger than for the corresponding time windows during intertrial crossings and during escapes. Thus, avoids are associated with more MUA in superior colliculus than intertrial crossings or escapes. Moreover, peak1 $(p<$ 0.01) MUA responses during escapes were significantly larger than during intertrial crossings, but this was not the case for peak2 $(p=0.9)$, peak3 $(p=0.5)$, or peak1-3 $(p=0.8)$ re- 
sponses. The larger trigeminotectal (peak1) MUA response during escapes than during intertrial crossings makes sense because the WCS is not present to drive trigeminotectal MUA during intertrial crossings. In conclusion, these results indicate that the ramped-up MUA during avoids is not caused by shuttling during avoids.

\section{Discussion}

Avoids are associated with the following neural correlates: (1) high pre-WCS firing rate in superior colliculus; (2) suppression (adaptation) of FP responses in barrel cortex and the related corticotectal activity (peak2 FP) in superior colliculus; (3) deemphasis of direct trigeminotectal inputs (peak1) in superior colliculus at WCS onset; and (4) a robust overall ramping up of the firing rate in superior colliculus.

We found that the spontaneous pre-WCS firing rate in superior colliculus is consistently larger during avoids than during escapes, indicating that an active (prepared) superior colliculus is more likely to detect the WCS than an inactive (nonprepared) superior colliculus. However, spontaneous pre-WCS FP activity in barrel cortex, which is quite sensitive to changes in behavioral states, was not different between avoids and escapes. Thus, the state of superior colliculus neural activity may determine whether a WCS is detected and avoided. By separating the late-session avoids from other trials, we attempted to get a glimpse of the neural activity that is associated with excellent performance in the avoidance task. It is worth noting that late-session avoids were associated with high pre-WCS MUA, but so were early-session escapes. The lack of difference when compared to early-session escapes may be attributable to the fact that animals are quite stressed during early-session escapes because they are unaware of how to cope with the task and are being shocked consistently. Thus, the enhanced firing rate in superior colliculus during earlysession escapes may reflect this state.

The results also showed that FP responses from WCS offset (when avoids occur and/or avoidance interval ends) in barrel cortex were more suppressed during avoids than during escapes. This reflected a stronger suppression (adaptation) of responses as the animal is about to avoid. Moreover, during late-session avoids, this effect was stronger so that both FP responses in barrel cortex (from offset and onset) and the related peak2 FP responses in superior colliculus are more suppressed than during earlysession escapes. In barrel cortex, stronger sensory suppression occurs in vigilant and attentive animals (Castro-Alamancos, 2004a,b) and leads to focusing of cortical representations during states of cortical activation (Castro-Alamancos, 2002; CastroAlamancos and Oldford, 2002). In superior colliculus, peak2 responses are driven by the barrel cortex and are also suppressed during cortical activation (Cohen et al., 2008). Thus, it is not surprising that effective performance in the task is associated with stronger sensory suppression in barrel cortex and the related corticotectal activity in superior colliculus.

Avoids were associated with a deemphasis of direct trigeminotectal inputs (peak1) in superior colliculus at WCS onset. Trigeminotectal responses are best measured during the onset of a high-frequency stimulus train because these responses depress with frequency (Cohen and Castro-Alamancos, 2007; Cohen et al., 2008). The fact that the firing rate during peak 1 responses from WCS onset were smaller during avoids (or late-session avoids) than during escapes indicates that superior colliculus becomes less responsive to the direct trigeminotectal input in well trained animals. This may be because the superior colliculus becomes reliant on higher-order processing centers to drive neural activity related to avoids. Regardless, the result seems to negate the possibility that avoidance learning is caused by a specific enhancement in the efficacy of direct trigeminotectal responses.

The importance of superior colliculus neural activity during active avoidance was particularly emphasized by the finding that the firing rate from WCS offset ramped up during avoids compared to either escapes or spontaneous shuttling (intertrial crossings). The enhancement of the firing rate in superior colliculus during avoids reflected an overall increase, and not the enhancement of a specific response time window (i.e., peak1, peak2, peak3) after each WCS stimulus in the trial. This conclusion was also confirmed by measuring evoked peak1 and peak2 FP responses in superior colliculus, neither of which was enhanced during avoids. These results indicate that neural activity in the intermediate layers of the superior colliculus robustly codes active avoidance behavior by ramping up the firing rate during avoids.

\section{Significance of the enhanced neural activity in superior colliculus during avoids}

An important finding of this study is that avoids are noticeably associated with a robust ramping up of the overall firing rate in superior colliculus. There are two obvious questions regarding this finding. One relates to how the enhanced firing rate is generated, which deals with the inputs to superior colliculus that may drive it. The other relates to the neural circuits that are driven by the enhanced superior colliculus output. Although an extensive discussion of input/output pathways of the superior colliculus and potential mechanisms is not possible here, we will mention a few possibilities.

\section{What neural circuits drive the activity in superior colliculus during avoids?}

One possibility is that direct trigeminotectal inputs drive the enhanced firing rate, but this appears unlikely because, as discussed above, trigeminotectal responses (peak1) tend to reduce their strength as the animal improves performance (late-session avoids). Another unlikely possibility is that the barrel cortex drives the activity in superior colliculus. The barrel cortex provides fast corticotectal activity driven by the WCS (Cohen et al., 2008), but we did not find a specific increase in the firing rate during the period when corticotectal activity arrives (peak2). Thus, the barrel cortex is unlikely the source driving the enhanced neural activity during avoids. Instead, a more likely possibility is that the ramping-up activity may be related to the basal ganglia because the superior colliculus forms loops through the basal ganglia (McHaffie et al., 2005; Redgrave and Gurney, 2006) and the basal ganglia may be involved in active avoidance behavior (Delacour et al., 1977; Chavez-Martinez et al., 1987; Packard and Knowlton, 2002). A suppression of the output of substantia nigra pars reticulata cells projecting to the superior colliculus leads through disinhibition to increased firing in superior colliculus (Chevalier et al., 1981; Jiang et al., 2003) and increases defensive reactions evoked by superior colliculus stimulation (Coimbra and Brandao, 1993; Brandao et al., 2003, 2005). Moreover, the superior colliculus output during avoids may drive cells in substantia nigra pars compacta to signal this event (Comoli et al., 2003; Dommett et al., 2005; Redgrave and Gurney, 2006) and influence the basal ganglia loop. Further exploration of the relationship between superior colliculus and substantia nigra during active avoidance is warranted. 
What circuits are driven by the enhanced superior colliculus activity and for what purpose?

The enhanced firing in superior colliculus may drive avoids. This could be accomplished through its outputs to the basal ganglia as mentioned above, through outputs to the amygdala, and/or through direct outputs to the brainstem. Regarding the involvement of the amygdala, cells of the paralaminar nuclei in the posterior thalamus that border the medial geniculate body project to the amygdala and are contacted by superior colliculus afferents (Linke et al., 1999), which provides a tecto-thalamo-amygdala pathway for the output to reach the amygdala during avoids. In pavlovian fear conditioning, which is different compared to active avoidance because the aversive outcome is not contingent on the animal's behavior, the CS is known to be relayed to the amygdala directly through the modality-specific sensory thalamus or indirectly via the cortex (Tischler and Davis, 1983; LeDoux et al., 1984; Davis and Whalen, 2001; Shi and Davis, 2001; Boatman and Kim, 2006; Cohen and Castro-Alamancos, 2007). However, the role of the amygdala in active avoidance is less evident; learning avoidance behavior may be dependent on the amygdala, but performance appears not to be (Roozendaal et al., 1993; Poremba and Gabriel, 1997, 1999).

A more plausible scenario is that through its brainstem projections, the superior colliculus drives avoids. The superior colliculus is well known to be involved in orienting, approach, and escape responses to stimuli from a wide range of modalities, including somatosensory, auditory, and visual (Sprague and Meikle, 1965; Schneider, 1969; Meredith and Stein, 1985; Sparks, 1986; Dean et al., 1989; Stein and Meredith, 1993; Stein, 1998). For example, looming stimuli (resembling a predator) can evoke escape responses in rats that are believed to be mediated by the superior colliculus (Dean et al., 1989; Westby et al., 1990). Stimulation of the superior colliculus produces defensive behaviors, of which escape responses are particularly relevant to active avoidance conditioning (Bandler et al., 1985; Dean et al., 1988, 1989; Brandao et al., 1994, 2003). These behaviors occur via two main descending pathways (predorsal bundle and ipsilateral efferent bundle) that originate in separate subregions of the deeper layers and can mediate approach and escape responses (Redgrave et al., 1987a,b, 1993; Westby et al., 1990; May, 2005). In particular, the ipsilateral efferent bundle is an ipsilateral descending projection with terminations in the periaqueductal gray, cuneiform nucleus, lateral pons, and ventral pontine/medullary reticular formation that can mediate escape behaviors. Thus, the ramping up of activity in superior colliculus may directly drive avoids through these output projections.

\section{References}

Ahissar E (1998) Temporal-code to rate-code conversion by neuronal phase-locked loops [letter]. Neural Comput 10:597-650.

Bandler R, Depaulis A, Vergnes M (1985) Identification of midbrain neurones mediating defensive behaviour in the rat by microinjections of excitatory amino acids. Behav Brain Res 15:107-119.

Boatman JA, Kim JJ (2006) A thalamo-cortico-amygdala pathway mediates auditory fear conditioning in the intact brain. Eur J Neurosci 24:894-900.

Bouton ME (2007) Learning and behavior. Sunderland: Sinauer.

Brandao ML, Cardoso SH, Melo LL, Motta V, Coimbra NC (1994) Neural substrate of defensive behavior in the midbrain tectum. Neurosci Biobehav Rev 18:339-346.

Brandao ML, Troncoso AC, Souza Silva MA, Huston JP (2003) The relevance of neuronal substrates of defense in the midbrain tectum to anxiety and stress: empirical and conceptual considerations. Eur J Pharmacol 463:225-233.

Brandao ML, Borelli KG, Nobre MJ, Santos JM, Albrechet-Souza L, Oliveira AR, Martinez RC (2005) Gabaergic regulation of the neural organiza- tion of fear in the midbrain tectum. Neurosci Biobehav Rev 29:1299-1311.

Castro-Alamancos MA (2002) Role of thalamocortical sensory suppression during arousal: focusing sensory inputs in neocortex. J Neurosci 22:9651-9655.

Castro-Alamancos MA (2004a) Absence of rapid sensory adaptation in neocortex during information processing states. Neuron 41:455-464.

Castro-Alamancos MA (2004b) Dynamics of sensory thalamocortical synaptic networks during information processing states. Prog Neurobiol 74:213-247.

Castro-Alamancos MA, Connors BW (1996) Short-term plasticity of a thalamocortical pathway dynamically modulated by behavioral state. Science 272:274-277.

Castro-Alamancos MA, Oldford E (2002) Cortical sensory suppression during arousal is due to the activity-dependent depression of thalamocortical synapses. J Physiol 541:319-331.

Chavez-Martinez ME, Reyes-Vazquez C, Brust-Carmona H (1987) Lever pressing and active avoidance conditioning after electrolytic lesions of the entopeduncular nucleus in cats. Brain Res Bull 18:279-284.

Chevalier G, Deniau JM, Thierry AM, Feger J (1981) The nigro-tectal pathway. An electrophysiological reinvestigation in the rat. Brain Res 213: 253-263.

Chung S, Li X, Nelson SB (2002) Short-term depression at thalamocortical synapses contributes to rapid adaptation of cortical sensory responses in vivo. Neuron 34:437-446.

Cohen JD, Castro-Alamancos MA (2007) Early sensory pathways for detection of fearful conditioned stimuli: tectal and thalamic relays. J Neurosci 27:7762-7776.

Cohen JD, Castro-Alamancos MA (2010) Detection of low salience whisker stimuli requires synergy of tectal and thalamic sensory relays. J Neurosci 30:2245-2256.

Cohen JD, Hirata A, Castro-Alamancos MA (2008) Vibrissa sensation in superior colliculus: wide-field sensitivity and state-dependent cortical feedback. J Neurosci 28:11205-11220.

Coimbra NC, Brandao ML (1993) GABAergic nigro-collicular pathways modulate the defensive behaviour elicited by midbrain tectum stimulation. Behav Brain Res 59:131-139.

Comoli E, Coizet V, Boyes J, Bolam JP, Canteras NS, Quirk RH, Overton PG, Redgrave P (2003) A direct projection from superior colliculus to substantia nigra for detecting salient visual events. Nature Neurosci 6:974-980.

Davis M, Whalen PJ (2001) The amygdala: vigilance and emotion. Mol Psychiatry 6:13-34.

Dean P, Mitchell IJ, Redgrave P (1988) Responses resembling defensive behaviour produced by microinjection of glutamate into superior colliculus of rats. Neuroscience 24:501-510.

Dean P, Redgrave P, Westby GW (1989) Event or emergency? Two response systems in the mammalian superior colliculus. Trends Neurosci 12:137-147.

Delacour J, Echavarria MT, Senault B, Houcine O (1977) Specificity of avoidance deficits produced by 6-hydroxydopamine lesions of the nigrostriatal system of the rat. J Comp Physiol Psychol 91:875-885.

Dommett E, Coizet V, Blaha CD, Martindale J, Lefebvre V, Walton N, Mayhew JE, Overton PG, Redgrave P (2005) How visual stimuli activate dopaminergic neurons at short latency. Science 307:1476-1479.

Garabedian CE, Jones SR, Merzenich MM, Dale A, Moore CI (2003) Bandpass response properties of rat SI neurons. J Neurophysiol 90:1379-1391.

Jensen O, Kaiser J, Lachaux JP (2007) Human gamma-frequency oscillations associated with attention and memory. Trends Neurosci 30:317-324.

Jiang H, Stein BE, McHaffie JG (2003) Opposing basal ganglia processes shape midbrain visuomotor activity bilaterally. Nature 423:982-986.

Khatri V, Hartings JA, Simons DJ (2004) Adaptation in thalamic barreloid and cortical barrel neurons to periodic whisker deflections varying in frequency and velocity. J Neurophysiol 92:3244-3254.

LeDoux JE, Sakaguchi A, Reis DJ (1984) Subcortical efferent projections of the medial geniculate nucleus mediate emotional responses conditioned to acoustic stimuli. J Neurosci 4:683-698.

Linke R, De Lima AD, Schwegler H, Pape HC (1999) Direct synaptic connections of axons from superior colliculus with identified thalamoamygdaloid projection neurons in the rat: possible substrates of a subcortical visual pathway to the amygdala. J Comp Neurol 403:158-170. 
May PJ (2005) The mammalian superior colliculus: laminar structure and connections. Prog Brain Res 151:321-378.

McHaffie JG, Kao CQ, Stein BE (1989) Nociceptive neurons in rat superior colliculus: response properties, topography, and functional implications. J Neurophysiol 62:510-525.

McHaffie JG, Stanford TR, Stein BE, Coizet V, Redgrave P (2005) Subcortical loops through the basal ganglia. Trends Neurosci 28:401-407.

Meredith MA, Stein BE (1985) Descending efferents from the superior colliculus relay integrated multisensory information. Science 227:657-659.

Moore CI (2004) Frequency-dependent processing in the vibrissa sensory system. J Neurophysiol 91:2390-2399.

Mowrer OH (1960) Learning theory and behavior. New York: Wiley.

Packard MG, Knowlton BJ (2002) Learning and memory functions of the basal ganglia. Annu Rev Neurosci 25:563-593.

Poremba A, Gabriel M (1997) Amygdalar lesions block discriminative avoidance learning and cingulothalamic training-induced neuronal plasticity in rabbits. J Neurosci 17:5237-5244.

Poremba A, Gabriel M (1999) Amygdala neurons mediate acquisition but not maintenance of instrumental avoidance behavior in rabbits. J Neurosci 19:9635-9641.

Redgrave P, Gurney K (2006) The short-latency dopamine signal: a role in discovering novel actions? Nat Rev Neurosci 7:967-975.

Redgrave P, Mitchell IJ, Dean P (1987a) Descending projections from the superior colliculus in rat: a study using orthograde transport of wheatgerm-agglutinin conjugated horseradish peroxidase. Exp Brain Res 68:147-167.

Redgrave P, Mitchell IJ, Dean P (1987b) Further evidence for segregated output channels from superior colliculus in rat: ipsilateral tecto-pontine and tecto-cuneiform projections have different cells of origin. Brain Res 413:170-174.

Redgrave P, Westby GW, Dean P (1993) Functional architecture of rodent superior colliculus: relevance of multiple output channels. Prog Brain Res 95:69-77.
Redgrave P, McHaffie JG, Stein BE (1996a) Nociceptive neurones in rat superior colliculus. I. Antidromic activation from the contralateral predorsal bundle. Exp Brain Res 109:185-196.

Redgrave P, Telford S, Wang S, McHaffie JG, Stein BE (1996b) Functional anatomy of nociceptive neurones in rat superior colliculus. Prog Brain Res 107:403-415.

Roozendaal B, Koolhaas JM, Bohus B (1993) The central amygdala is involved in conditioning but not in retention of active and passive shock avoidance in male rats. Behav Neural Biol 59:143-149.

Schneider GE (1969) Two visual systems. Science 163:895-902.

Schroeder CE, Lakatos P (2009) Low-frequency neuronal oscillations as instruments of sensory selection. Trends Neurosci 32:9-18.

Shi C, Davis M (2001) Visual pathways involved in fear conditioning measured with fear-potentiated startle: behavioral and anatomic studies. J Neurosci 21:9844-9855.

Sparks DL (1986) Translation of sensory signals into commands for control of saccadic eye movements: role of primate superior colliculus. Physiol Rev 66:118-171.

Sprague JM, Meikle TH Jr (1965) The role of the superior colliculus in visually guided behavior. Exp Neurol 11:115-146.

Stein BE (1998) Neural mechanisms for synthesizing sensory information and producing adaptive behaviors. Exp Brain Res 123:124-135.

Stein BE, Dixon JP (1979) Properties of superior colliculus neurons in the golden hamster. J Comp Neurol 183:269-284.

Stein BE, Meredith MA (1993) The merging of the senses. Cambridge, MA: MIT.

Tischler MD, Davis M (1983) A visual pathway that mediates fearconditioned enhancement of acoustic startle. Brain Res 276:55-71.

Vanderwolf CH (1988) Cerebral activity and behavior: control by central cholinergic and serotonergic systems. Int Rev Neurobiol 30:225-340.

Westby GW, Keay KA, Redgrave P, Dean P, Bannister M (1990) Output pathways from the rat superior colliculus mediating approach and avoidance have different sensory properties. Exp Brain Res 81:626-638. 